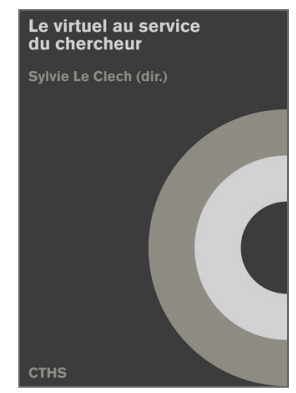

Sylvie Le Clech (dir.)

Le virtuel au service du chercheur

Éditions du Comité des travaux historiques et scientifiques

\title{
L'édition numérique de la correspondance de François Guizot et de la princesse de Lieven (1836-1856) sur la plateforme EMAN : Association François-Guizot - ITEM (CNRS/ENS)
}

\section{Marie Dupond}

DOI : $10.4000 /$ books.cths. 15318

Éditeur : Éditions du Comité des travaux historiques et scientifiques

Lieu d'édition : Éditions du Comité des travaux historiques et scientifiques

Année d'édition : 2021

Date de mise en ligne : 21 juin 2021

Collection : Actes des congrès nationaux des sociétés historiques et scientifiques

EAN électronique : 9782735509300

\section{Cbooks}

http://books.openedition.org

\section{Référence électronique}

DUPOND, Marie. L'édition numérique de la correspondance de François Guizot et de la princesse de Lieven (1836-1856) sur la plateforme EMAN : Association François-Guizot - ITEM (CNRS/ENS) In : Le virtuel au service du chercheur [en ligne]. Paris : Éditions du Comité des travaux historiques et scientifiques, 2021 (généré le 24 juin 2021). Disponible sur Internet : <http://books.openedition.org/cths/15318>. ISBN : 9782735509300. DOI : https://doi.org/10.4000/books.cths.15318. 


\title{
L'édition numérique de la correspondance de François Guizot et de la princesse de Lieven (1836-1856) sur la plateforme EMAN : Association François- Guizot - ITEM (CNRS/ENS)
}

\author{
Marie Dupond
}

1 Une des premières difficultés à laquelle se confronte l'éditeur de correspondance est la constitution et l'inventaire d'un corpus de lettres. C'est justement la recommandation décisive donnée en 1984 dans le Petit guide de l'éditeur de correspondance: toujours considérer l'inventaire d'un corpus "comme incomplet et susceptible d'amélioration ${ }^{1} »$. Ainsi, on saisit d'emblée l'horizon que le numérique a ouvert aux éditeurs de correspondance en permettant l'enrichissement et la modification des corpus épistolaires ${ }^{2}$. Depuis le début des années 2000, le développement de la numérisation a stimulé les entreprises éditoriales au sein de dispositifs informatiques ${ }^{3}$. On a d'abord parlé d'éditions électroniques, puis d'éditions numériques en associant de plus en plus l'édition électronique au milieu numérique de sa publication ${ }^{4}$. Mais si le numérique a favorisé le développement des entreprises éditoriales de la part de chercheurs, il a aussi conduit le chercheur-éditeur de correspondances à inscrire ses pratiques et sa démarche dans les réseaux de questionnements scientifiques et épistémologiques qui définissent le champ pluridisciplinaire des humanités numériques ${ }^{5}$.

2 Le traitement éditorial et l'étude critique des sources sont désormais associés à une dimension technologique. Cela suscite une dynamique collaborative, interprofessionnelle et interdisciplinaire entre différents acteurs: chercheurs, ingénieurs, archivistes et documentalistes. Ainsi, la démarche éditoriale doit être 
associée à une démarche critique et méthodologique qui considère tant le dispositif technique que les enjeux scientifiques et patrimoniaux des entreprises éditoriales.

Le traitement éditorial d'un corpus documentaire ou scientifique en milieu numérique se nomme «éditorialisation ». L'édition numérique en est le résultat. Dès sa conception, le processus éditorial est associé à sa médiatisation et aux dispositifs techniques de sa réalisation. Ce terme d'« éditorialisation» est un anglicisme, qui permet de saisir l'inscription de l'acte éditorial dans un réseau de conditions et d'acteurs. Il met en évidence la coordination d'un dispositif technique à des pratiques scientifiques, en envisageant un développement structuré du contenu pour répondre à des objectifs tant scientifiques que culturels et patrimoniaux ${ }^{6}$.

Le projet d'éditorialisation de la correspondance entre François Guizot (1787-1874) et Dorothée van Benckendorff, princesse de Lieven (1785-1857), sur la plateforme EMAN (Institut des textes et manuscrits modernes, CNRS/ENS), est financé par l'Association François-Guizot. L'Association a pour but de faire connaître l'ensemble de l'œuvre, notamment intellectuelle et politique, de François Guizot et de sa famille ${ }^{7}$. Elle mène ainsi plusieurs actions de médiation et de valorisation de ce patrimoine. Récemment, l'Association a donc décidé d'éditer le corpus Guizot-Lieven en inscrivant ce projet dans une dynamique scientifique et innovante. C'est dans ce cadre que j'ai été chargée du projet d'éditorialisation de leur correspondance croisée, de 1836 à 1856 . Il s'agit d'appliquer à ce corpus un traitement éditorial qui répond aux principes archivistiques et scientifiques déterminés par l'environnement numérique. Pour ce faire, on lui applique le schéma éditorial établi sur EMAN pour l'édition de la correspondance de Gaspard Monge ${ }^{8}$, ainsi que les principes constitués au sein du groupe de travail Correspondance du consortium CAHIER (Huma-Num 2016-2018) ${ }^{9}$ et au cours d'une recherche postdoctorale ${ }^{10}$.

5 En présentant des éléments de l'histoire de ce corpus et de sa transmission, je voudrais souligner les enjeux scientifiques et méthodologiques de son éditorialisation, indiquer les principes établis et les phases du traitement éditorial déterminées par une entreprise au sein de la plateforme EMAN $^{11}$.

\section{Enjeux éditoriaux, histoire et nature du corpus}

6 Pour établir des principes d'édition d'un corpus et en saisir les enjeux, il faut considérer son histoire éditoriale et sa nature. Tout d'abord, c'est un corpus volumineux, ils s'écrivent chaque jour.

7 Dorothée écrit à François, en septembre 1840 :

" Je vous ai écrit au moment de me coucher, je vous écris à mon lever. Toujours, toujours penser à vous. Vous parler ou vous écrire, selon que le ciel ordonne que ce soit l'un ou l'autre, voilà comme se passera ma vie ${ }^{12}$.»

François écrit à Dorothée, en mars 1840 :

"Vous trouvez mes lettres trop courtes, tant mieux. Elles sont longues pourtant. Mesurez mon écriture. Vous verrez qu'une de mes pages en tient deux des vôtres. Mais trouvez-les toujours trop courtes. Certainement non, nous ne nous disons pas tout, et c'est l'immense ennui de l'absence. Il n'y a pas moyen que je vous dise tout ce qui me traverse le cœur et l'esprit en vous écrivant ${ }^{13}$. »

9 Cette correspondance croisée est constituée de plus de 4000 lettres échangées pendant 20 ans, et elle est aussi bien amoureuse que politique et diplomatique. Leurs premiers 
échanges commencent en 1836. Dorothée, princesse de Lieven, femme d'un diplomate russe, a 51 ans et François Guizot, ministre de l'Instruction publique, veuf, a 50 ans. Si leur intimité se noue à partir de l'expérience commune du deuil d'un enfant, leur rencontre a lieu dans des espaces sociaux et politiques qu'ils partagent et se développe rapidement autour de leur vif intérêt pour la chose publique et de leur positionnement respectif au sein du réseau diplomatique de l'Europe du XIX ${ }^{\mathrm{e}}$ siècle. Dorothée et François ont une intense activité épistolaire et ont conscience des usages de cette pratique et de ses fonctions de communication, de diffusion et de croisement des informations. Laurent Theis souligne que leur rencontre s'inscrit dans un réseau de conditions socioculturelles qui, depuis la révolution de 1830, rend possible le binôme d'un bourgeois au pouvoir par son seul mérite et d'une grande aristocrate qui trouve à s'investir dans un monde nouveau. On pense aussi à Madame de Staël et Napoléon, Madame de Krüdener et le tsar Alexandre, la duchesse de Duras et Chateaubriand ou encore à Dorothée de Dino et Talleyrand. Le réseau de Dorothée de Lieven est très étendu : elle a établi autour d'elle une société paneuropéenne qui convient particulièrement à François Guizot et lui permet de mettre en perspective les rapports entre histoire, identité nationale et européenne. Dans ses lettres, Dorothée de Lieven offre à François Guizot un regard riche et complexe sur la politique française dans une dimension internationale.

Cela est manifeste lorsque Dorothée écrit à François, en mars 1840 :

"Voilà les observations de la diplomatie. Tout ceci aujourd'hui fait un spectacle curieux à observer ${ }^{14}$. "

11 Il lui répond:

«Vous m'en apprenez plus que tous les autres. Ils dissertent. Vous racontez ${ }^{15}$.»

Un des enjeux de l'édition est de mieux connaitre le fonctionnement des salons, mais aussi celui des correspondances comme espace et pratique d'action publique et sociale des femmes au XIXe siècle. Le privé se mêle au public en structurant et animant des réseaux politiques, sociaux et culturels. Cela permet de saisir la nécessité d'envisager ensemble la correspondance privée et publique, de développer des liens avec d'autres types de documents pour soulever la question des rapports entre action publique et œuvre intellectuelle, en favorisant ainsi des approches interdisciplinaires de la correspondance comme genre transdisciplinaire. On peut en effet observer comment l'usage de méthodes et d'outils d'autres disciplines peut renouveler les questionnements et manifester des enjeux historiographiques et biographiques.

Comme les auteurs, il faut distinguer au sein des lettres deux types de discours épistolaire :

1. Le journal, où est développé le récit de leurs activités, de leurs rencontres et conversations, mais aussi des événements politiques et diplomatiques en France, en Europe et dans le monde. Mots-clés : réseau social et politique, politique (France), politique (Angleterre), politique (Russie), politique (internationale), diplomatie, etc.

2. La lettre est l'espace du discours intime et autobiographique. C'est aussi celui du discours réflexif et théorique sur les arts, la politique, l'histoire, etc., ainsi que sur leur parcours personnel. Mots-clés: autoportrait, portrait, discours du for intérieur, discours autobiographique, politique, histoire, poésie, littérature, musique, etc.

Par exemple, François écrit à Dorothée, le 29 juillet 1837 du Val-Richer :

« [...] à la voir du dehors, j'ai mené une vie toute d'action, toute vouée au public, qui a dû, qui doit paraître surtout ambitieuse, personnelle, presque sévère. Et en effet, j'ai pris et je prends, à ce qui m'a occupé, aux études, aux affaires, aux luttes 
politiques, un grand, très grand intérêt. Je m'y suis adonné et m'y adonne avec grand plaisir comme à un emploi naturel et satisfaisant de moi-même. J'y désire vivement l'éclat et le succès. [...] Et pourtant [...] là n'est point tout, là n'a jamais été ma véritable vie, de là je n'ai jamais reçu aucune émotion, aucune satisfaction qui atteignit jusqu'au fond de mon âme, de là n'est jamais venu le sentiment du bonheur! Le bonheur, Madame, le bonheur qui pénètre partout dans l'âme, qui la remplit et l'assouvit toute entière [...] est quelque chose, de bien supérieur à tout ce que la vie publique peut donner ${ }^{16}$. »

François Guizot aime la correspondance, il aime la lire et la relire. Il aime aussi l'écrire. Ce genre lui permet d'allier les descriptions, les récits, les portraits, mais aussi les discours autobiographiques, historiques et théoriques. Il a aussi une conscience d'historien, et connaît la fécondité de sa correspondance avec Dorothée comme source. En 1833 et 1834, François Guizot a permis la création de la Société d'histoire de France et du Comité des travaux historiques et scientifiques. En institutionnalisant la connaissance et la diffusion du passé, il permet alors au mouvement historiographique, archivistique et archéologique de passer de l'amateurisme éclairé au savoir scientifique. Il conserve intégralement leur correspondance et il fait transcrire par son secrétaire Hennequin ${ }^{17}$ les lettres de 1837 à 1843 sans aucune intervention sur le texte. C'est un moment d'analyse et de recul, tout en constituant un moment décisif pour son positionnement public. La transcription intégrale manuscrite est rassemblée dans dix volumes reliés de maroquin vert. François Guizot avait pour pratique de rassembler les correspondances échangées avec les femmes qu'il a aimées. Il confie à Dorothée au tout début de leur relation - ce qu'elle appelle avec pudeur les deux romans - sa correspondance avec Pauline de Meulan et Eliza Dillon. François Guizot a veillé luimême à conserver et établir un corpus de sources d'un moment d'élaboration et de réflexion sur les motifs de son action publique et ses principes politiques, mais aussi de son œuvre historique.

De 1963 à 1964 paraît l'édition de Jacques Naville et Jean Schlumberger en trois volumes d'extraits de la correspondance de 1837-1846 ${ }^{18}$. Jusqu'en 1959, la correspondance est conservée dans les archives familiales du Val-Richer. En 1959, lors de la préparation de l'édition partielle de la correspondance, le corpus intégral est déposé aux Archives nationales et une copie microfilmée des lettres est réalisée. En 2017, la numérisation des copies microfilmées dans la perspective d'une édition numérique a été la première phase du projet. C'est bien dans ce premier moment technique de virtualisation par la numérisation de l'objet physique que le document entre dans le processus d'éditorialisation et de patrimonialisation.

L'édition de 1963 ne rassemble que de larges extraits des lettres de 1837 à 1846 . L'objectif éditorial du projet EMAN est de compléter le corpus de 1847 à 1856 et de donner accès à l'intégralité des lettres de Dorothée et François, tant aux autographes qu'à un discours épistolaire décrit, documenté, indexé et transcrit. Le projet est ambitieux, puisque 11000 feuillets numérisés restent à éditorialiser. L'édition cherche à valoriser ce corpus en l'ouvrant à de nouvelles investigations scientifiques depuis d'autres champs disciplinaires, en exploitant les ressources documentaires établies par Laurent Theis et rassemblées dans les contenus thématiques et biographiques du site de l'Association François-Guizot. 


\section{Gestion de projet et traitement éditorial} Un premier objectif a été atteint avec l'ouverture du site en janvier $2020^{19}$. Cette édition est envisagée comme une bibliothèque numérique structurée en collections. Sont publiés 410 lettres, plus de 2400 folios éditorialisés et transcrits, au sein de 8 collections ${ }^{20}$. Le dispositif numérique permet un accès à des sources patrimoniales et scientifiques en cours d'élaboration. De nouveaux corpus seront régulièrement publiés et la documentation du matériau enrichie.

19 L'établissement de principes et objectifs éditoriaux précis est nécessaire pour déterminer des phases successives de réalisation, dans une perspective méthodologique et pragmatique. La description raisonnée des modalités du traitement éditorial doit être inscrite sur une ligne de temps. Le premier principe à considérer est celui de faisabilité, en envisageant les ressources humaines et financières.

L'objectif de cette édition est de donner à lire en réseau, mais aussi d'explorer un corpus de correspondance. Un premier schéma éditorial EMAN a été réalisé sur un corpus test de vingt lettres. Cela a permis de tester la méthodologie du traitement éditorial. L'environnement numérique a ses exigences. Les principes d'éditorialisation doivent permettre l'établissement de réseaux de relations entre unité documentaire, collection, corpus, à partir non seulement des données d'identification, de description, de documentation et d'indexation, mais aussi de transcription et de mise en relation. Il faut déterminer des phases de réalisation du projet éditorial pour systématiser et mettre en œuvre ces pratiques qui se réalisent au cours la mise en données.

21 EMAN est une plateforme de publication et d'investigation scientifique en milieu numérique, coordonnée par un ingénieur-éditeur et animée par les acteurs des projets. La plateforme repose sur une culture technique et scientifique issue du partage d'un dispositif technique générique et de son usage interdisciplinaire et interprofessionnel, à partir du système de gestion de contenu Omeka ${ }^{21}$. Cet outil offre un modèle de détermination et de structuration des données adéquates pour servir des préoccupations scientifiques multidisciplinaires et des objectifs culturels, patrimoniaux et pédagogiques ${ }^{22}$. Ces données sont interopérables et exportables afin d'assurer tant leur pérennité que leur exploitation au sein d'autres protocoles de recherche avec un outillage technique différent.

Il faut alors considérer comment, dans un environnement numérique, les rapports entre archivistique, édition et recherche, sont décisifs dans la production de données interopérables pérennes, «moissonnables » et exportables et dans l'élaboration d'une structuration économique ${ }^{23}$. Au cours de l'éditorialisation, une dynamique des pratiques archivistiques, éditoriales et scientifiques s'établit. L'expérience des bibliothèques et des centres d'archives en tant que référentiels est décisive pour la production de données pertinentes pour authentifier, identifier et décrire une diversité de document. Cela est nécessaire pour réaliser la mise en relation de document non seulement au sein d'un corpus, mais parmi l'ensemble des ressources archivistiques publiées en environnement numérique. De même, la valorisation d'un corpus par la recherche scientifique se produit lors de la coordination entre identification et description pour manifester des enjeux éditoriaux. La recherche est nécessaire pour la structuration du corpus et la détermination d'entrées thématiques et de parcours problématisés et documentés. 


\section{Établissement d'un inventaire comparé et préparation des données d'identification, de description et d'indexation}

Pour concevoir une première phase de traitement éditorial et préparer les données, une base est établie à partir d'un inventaire comparé des manuscrits du fonds conservé aux Archives nationales, des transcriptions manuscrites, des copies microfilmées et numérisées et des lettres éditées par Jean Schlumberger. Sont associés à chaque lettre les fichiers images correspondants. Les données d'identification, de description matérielle et circonstancielle et d'indexation thématique sont établies au sein d'un tableur. Ainsi, la création de notices pour chaque lettre et l'import des données structurées associées peuvent être effectués en masse par l'ingénieur.

\section{Principes de documentation, de description, d'indexation et de navigation}

L'appareillage du corpus ne s'effectue pas de la même façon que dans une édition papier. Ainsi, l'ensemble des informations indiquées sous la forme de notes de bas de page sont distribuées sur EMAN, en données structurées en champs Dublin Core, en données "personnalisées", en mise en relation ${ }^{24}$. Cette structure est proposée par le système Omeka, visant la correspondance avec les normes archivistiques et scientifiques.

La mise en données - c'est-à-dire la détermination et l'attribution des caractéristiques d'une lettre - doit être conçue pour permettre tant un traitement qualitatif que quantitatif. Ainsi, cela permet de ménager, dans une perspective dynamique, des phases de lecture humaine et d'approche computationnelle. Cela doit conduire à une précision des pratiques de déterminations de données pertinentes, afin de ne pas seulement viser des objectifs de mise à disposition, mais aussi de préparer le corpus à des investigations scientifiques en offrant la possibilité de croiser dans le détail des corpus volumineux ${ }^{25}$.

Au sein des champs déterminés sont indiqués les éléments de la description matérielle et circonstancielle (date, lieu, heure, etc.). L'identification des personnes, des lieux et des événements permet une exploitation computationnelle et une mise en réseau de la correspondance dans un ensemble de sources.

Le corpus de correspondance est structuré en collections et sous-collections. La présentation de ces dernières permet de documenter le corpus en introduisant des éléments biographiques et les sujets traités dans les lettres.

L'indexation thématique offre des entrées dans l'œuvre intellectuelle et publique de François Guizot par mots-clés. Elle est réalisée à partir des sujets traités au sein de la correspondance dans une perspective biographique. La navigation entre les lettres est ainsi permise par ces mots-clés, mais aussi par la mise en relation.

Pour la navigation entre lettres par mise en relation, un traitement scientifique est nécessaire - notamment pour la reconstitution du corpus croisé. Il faut aussi considérer la nature des relations entre les lettres. En environnement numérique et avec les caractéristiques du corpus Guizot-Lieven, cinq types de relations ont été identifiés :

- Cette lettre X est écrite avant la lettre A. 
- Cette lettre X est écrite après la lettre B.

- Cette lettre X est écrite le même jour que la lettre C.

- Cette lettre $\mathrm{X}$ répond à la lettre $\mathrm{D}$.

- La lettre $\mathrm{E}$ répond à cette lettre $\mathrm{X}$. où l'on a accès tant aux transcriptions qu'à l'image du manuscrit autographe. La normalisation de l'orthographe permet une recherche plein texte à tous les utilisateurs. La transcription doit répondre aux principes d'intelligibilité, d'accessibilité et d'« interrogeabilité ».

trique de transcription a ses principes mais aussi ses outils. Ici, la transcription est réalisée avec Transkribus, un outil de reconnaissance de caractères manuscrits en faisant usage de l'intelligence artificielle ${ }^{26}$. Mais un protocole doit être suivi. En effet, il faut d'abord préparer un matériau pour utiliser les outils Transkribus. La première phase de transcription intégrale de cent folios par auteur a été réalisée en ayant recours aux transcriptions de l'édition de 1963, réalisées pour la période 1836-1846 grâce aux procédés d'OCR (optical character recognition) associés à une relecture comparée avec les autographes et à une transcription manuelle pour compléter et obtenir le texte intégral. Les images et les textes associés sont ensuite importés sur le serveur Transkribus. Suit une phase d'apprentissage automatique, à l'issue de laquelle un modèle par scripteur est créé. Une phase de relecture et correction des transcriptions automatisées sur l'interface Transkribus, folio par folio, permet un perfectionnement des modèles. Pour le traitement du reste des folios, il faut successivement faire usage des outils de reconnaissance de zones de texte et de caractères. Enfin, lors de la relecture et de l'intégration des transcriptions au sein des notices de la plateforme EMAN, la question traditionnelle du choix des principes entre fidélité et lisibilité se pose à nouveau.

L'éditorialisation d'un corpus d'archives consiste en la coordination de pratiques éditoriales, archivistiques et scientifiques identifiées et mises en œuvre au sein d'un dispositif technique en milieu numérique. L'établissement de principes d'édition raisonnés est décisif tant pour l'usager que pour le concepteur et le contributeur. Mais il faudrait aussi considérer des principes d'usages des travaux d'éditorialisation des correspondances en veillant à la valorisation et à l'évaluation de telles entreprises comme production scientifique.

L'édition numérique constitue un espace de valorisation de la recherche, de ses méthodes et de ses résultats, offrant un accès plus précis, plus clair et plus direct au patrimoine culturel et scientifique. Les acteurs d'éditorialisation sont des acteurs de médiation, de médiatisation et de patrimonialisation. Il y a dans cette triple mission un enjeu épistémologique et historiographique, mais aussi culturel ${ }^{27}$. 


\section{BIBLIOGRAPHIE}

AMBROISE-RENDU Anne-Claude et OLIVESI Stéphane, « Du patrimoine à la patrimonialisation : perspectives critiques », Diogène, vol. CCLVIII-CCLIX-CCLX, nos 2-3-4, 2017, p. 265-279. [URL : https://www.cairn.info/revue-diogene-2017-2-page-265.htmoir]

CLAVERT Frédéric, « Lecture des sources historiennes à l'ère numérique ", L'histoire contemporaine à l'ère du numérique : humanités et études numériques en histoire contemporaine, carnet de recherche, éd. numérique, 2012. [URL : https://histnum.hypotheses.org/1061]

Correspondance croisée entre François Guizot et Dorothée de Benckendorff, princesse de Lieven (1836-1857), Dupond Marie (éd.), projet EMAN, Association François-Guizot - Institut des textes et manuscrits modernes (ITEM), CNRS-ENS. [URL : http://eman-archives.org/guizot-lieven]

Duchatelet B. et LE GUILlou L., Petit guide de l'éditeur de correspondances (XIX ${ }^{e}$-XX ${ }^{e}$ siècles), Brest, Centre brestois du Greco 53 du CNRS, 1986.

DUPOND Marie, « Des principes d'édition aux principes d'éditorialisation ». [URL : http://emanarchives.org/monge/des-principes-ddition-aux-principes-dditorialisation]

DUPOND Marie et FERJOUX Céline, « Usages des patrimoines numérisés : un réseau interdisciplinaire et interinstitutionnel. Mise en réseau d'objectifs, d'expériences, de pratiques et de méthodes », rapport postdoctoral, Paris, université Sorbonne Paris-Cité, 2017. [URL : http://udpn.fr/ spip.php?article288]

Épistolaire, $\mathrm{n}^{\circ}$ 33, Éditer les correspondances, Paris, Honoré Champion, 2007.

IDMHAND Fatiha et GALLERON Ioana « Guide pour la FAIRisation des données des corpus d'auteurs préparé par Fatiha Idmhand et Ioana Galleron pour le [Groupe de travail Data_Cahier] », rapport de recherche, TGIR Huma-Num, 2020. [URL : 〈halshs-02889777)]

JAQUET Christophe, « Métadonnées et Dublin Core », blog Open Web, 2010 [2003]. [URL : https:// openweb.eu.org/articles/dublin_core]

Lettres de François Guizot et de la princesse de Lieven (1837-1846), Schlumberger Jean et Naville Jacques (éd.), Paris, Mercure de France, 1963 (3 vol.).

MOUNIER Pierre, Les humanités numériques : une histoire critique, Paris, Éditions de la Maison des sciences de l'homme, 2018.

VITALI-ROSATI Marcello, « Qu'est-ce que l'éditorialisation? », Sens public, éd. numérique, 2016.

[URL : http://sens-public.org/article1184.html]

\section{NOTES}

1. B. Duchatelet et L. Le Guillou, Petit guide de l'éditeur de correspondances [...], p. 2.

2. Sur l'adéquation entre la correspondance et l'environnement numérique, voir M. Dupond, « Des principes d'édition aux principes d'éditorialisation ».

3. En 2004, l'édition de la correspondance d'André-Marie Ampère au sein du site Ampère et l'histoire de l'électricité en est un exemple. [URL: http://www.ampere.cnrs.fr/ correspondance/] La même année, le projet d'édition papier de la correspondance de Vincent Van Gogh se transforme en projet numérique. [URL: http:// 
vangoghletters.org/vg/about_1.html] En 2009 est publié l'inventaire de la correspondance de D'Alembert. [URL: http://dalembert.academie-sciences.fr/ Correspondance/] En 2007, la question est traitée dans la revue Épistolaire qui consacre un numéro à l'édition de correspondance, voir Épistolaire, $\mathrm{n}^{\circ} 33$.

4. La multiplication de ces nouvelles pratiques éditoriales de corpus épistolaires dans les champs des humanités a conduit en 2011 à la création d'un groupe de travail Correspondance au sein du consortium CAHIER (Corpus d'auteurs pour les humanités : informatisation, édition, recherche) au sein de la très grande infrastructure de recherche (TGIR) Huma-Num. [URL : https://cahier.hypotheses.org/activites/groupecorrespondance]

5. Pour une perspective historique et critique sur les humanités numériques, voir P. Mounier, Les humanités numériques [...].

6. La définition de l'éditorialisation établie par Marcello Vitali-Rosati permet d'en distinguer les différents éléments et pratiques pour mieux interroger les rapports entre forme et contenu, entre théorie, pratique et technique: "On peut définir l'éditorialisation comme un ensemble d'appareils techniques (le réseau, les serveurs, les plateformes, les CMS, les algorithmes des moteurs de recherche), de structures (l'hypertexte, le multimédia, les métadonnées), de pratiques (l'annotation, les commentaires, les recommandations via les réseaux sociaux) qui permet de produire et d'organiser un contenu sur le Web.»; voir M. Vitali-Rosati, "Qu'est-ce que l'éditorialisation?». Il ne manque pas de souligner qu'éditer avec les technologies numériques a un impact tant sur le contenu que sur la manière d'y accéder, de l'exploiter et de l'utiliser.

7. https://www.guizot.com/fr/

8. G. Monge "La correspondance inédite du géomètre Gaspard Monge (1746-1818) ", Dupond M. (éd.). [URL : http://eman-archives.org/monge/]

9. Groupe de travail Correspondance du consortium CAHIER, «L'édition numérique de correspondance : guide méthodologique ». [URL : https://cahier.hypotheses.org/guidecorrespondance]

10. M. Dupond, « Des principes d'édition aux principes d'éditorialisation ».

11. La plateforme EMAN, créée en 2015, rassemble désormais neuf projets d'éditorialisation de correspondance. Un groupe de travail Correspondance est animé à partir d'un séminaire en ligne : «L'épistolaire à l'ère du numérique : entre perspectives historiques et innovations technologiques ». [URL : http://www.item.ens.fr/epistolaireet-numerique]

12. 418. Poix, jeudi 10 septembre 1840, Dorothée de Lieven à François Guizot. [URL : http:// eman-archives.org/Guizot-Lieven/items/show/445]

13. 324. Londres, dimanche 15 mars 1840, François Guizot à Dorothée de Lieven. [URL : http:// eman-archives.org/Guizot-Lieven/items/show/192]

14. 322. Paris, mardi 10 mars 1840, Dorothée de Lieven à François Guizot. [URL : http://emanarchives.org/Guizot-Lieven/items/show/15]

15. 324. Londres, dimanche 15 mars 1840, François Guizot à Dorothée de Lieven. [URL : http:// eman-archives.org/Guizot-Lieven/items/show/192]

16. 13. Val-Richer, samedi 29 juillet 1837, François Guizot à Dorothée de Lieven. [URL : http:// eman-archives.org/Guizot-Lieven/items/show/899] 
17. Sans doute à partir de 1844 .

18. Lettres de François Guizot et de la princesse de Lieven (1837-1846), éditées pour la première fois de 1963 à 1964 par J. Schlumberger et J. Naville. Une collection sur la plateforme EMAN «L'édition des lettres de François Guizot et de la princesse de Lieven » est consacrée au matériau critique disponible dans cette première édition.

19. http://eman-archives.org/Guizot-Lieven/

20. http://eman-archives.org/Guizot-Lieven/collections/show/7

21. Un système de gestion de contenu est aussi appelé CMS en anglais (content management system). Le système Omeka, lancé en 2008, cherche à apporter des solutions de publication de contenu et de valorisation de collections aux institutions patrimoniales et scientifiques. [URL: https://omeka.org/about/project/] De très nombreux projets éditoriaux ont été réalisés en utilisant Omeka. Un état des lieux en France a été réalisé lors des Journées Omeka France en octobre 2020. [URL : https:// omeka.sciencesconf.org/resource/page/id/3]

22. Le milieu numérique conduit à envisager ensemble et à coordonner tant des usages que des objectifs scientifiques, patrimoniaux et culturels. C'est un des premiers résultats de l'étude des usages de patrimoines numérisés. Sur la question de la numérisation du ou des patrimoines et des enjeux du numérique dans la numérisation et l'éditorialisation de corpus, voir le rapport M. Dupond et C. Ferjoux, «Usages des patrimoines numérisés [...] ».

23. Cette question si décisive a conduit à l'établissement d'un groupe de travail autour de la «FAIRisation». Son enjeu est clairement défini par le groupe de travail du consortium CAHIER: «Les principes FAIR (Findability, Accessibility, Interoperability, and Reusability) définissent un ensemble minimal de principes qui permettent aux machines et aux humains de trouver, d'accéder, d'inter-opérer et de réutiliser les données et métadonnées de recherche. Les principes FAIR doivent être considérés comme des bonnes pratiques destinées à faciliter la réutilisation des données et des résultats de la recherche. » Voir F. Idmhand et I. Galleron, « Guide pour la FAIRisation des données des corpus d'auteurs [...]»"

24. Le schéma de métadonnées Dublin Core a été établi par des équipes impliquées dans la sémantique sur le Web lors d'un atelier à Dublin (Ohio) en 1995. Ce jeu de métadonnées a été surnommé Dublin Core Metadata. Parmi les propriétés cataloguées par la Dublin Core Metadata Initiative (DCMI), quinze ont été normalisées. Voir C. Jaquet, « Métadonnées et Dublin Core ».

25. F. Clavert, « Lecture des sources historiennes à l'ère numérique ».

26. Cet outil issu d'un projet scientifique a été testé gratuitement. Mais depuis octobre 2020, son usage est devenu payant. [URL : https://readcoop.eu/transkribus/? $\mathrm{sc}=$ Transkribus]

27. L'étude des usages des corpus numérisés a permis d'identifier les processus à l'œuvre, notamment la patrimonialisation et la médiatisation performées au cours du geste scientifique de définition et d'éditorialisation d'un corpus. Voir M. Dupond et C. Ferjoux, «Usages des patrimoines numérisés [...] ». En 2018 et 2019, le processus de patrimonialisation a été l'objet du séminaire du Centre d'histoire culturelle des sociétés contemporaines, de la Maison des sciences humaines Paris-Saclay et du comité d'histoire du ministère de la Culture: «Patrimoine et patrimonialisation: les inventions du capital historique ( $\mathrm{xIX}^{\mathrm{e}}-\mathrm{XxI}^{\mathrm{e}}$ siècles) », animé par Anne-Claude Ambroise- 
Rendu et Stéphane Olivesi. Voir A.-C. Ambroise-Rendu et S. Olivesi, « Du patrimoine à la patrimonialisation : perspectives critiques ».

\section{RÉSUMÉS}

Éditer les 4000 lettres de 1836 à 1856 de la correspondance croisée entre l'historien et homme d'État François Guizot (1787-1874), et une figure féminine du monde diplomatique européen du XIX ${ }^{\mathrm{e}}$ siècle, Dorothée van Benckendorff, princesse de Lieven (1785-1857), c'est éditer une partie des archives privées d'un acteur politique et intellectuel engagé dans le domaine public. Le projet d'éditorialisation de la correspondance Guizot-Lieven sur la plateforme EMAN (ITEM, CNRS/ENS) s'inscrit dans une perspective méthodologique et scientifique. En considérant l'histoire et la nature de ce corpus de correspondance, le traitement éditorial répond aux principes déterminés par les modalités techniques de l'environnement numérique, en distinguant les étapes du processus éditorial et les pratiques scientifiques et archivistiques mises en œuvre, et en posant la question des rapports entre édition et recherche, et entre édition et médiation au service de la valorisation et de la patrimonialisation.

\section{AUTEUR}

\section{MARIE DUPOND}

Docteur en épistémologie, histoire des sciences et des techniques, éditrice sur la plateforme EMAN (ITEM), CNRS/ENS, chargée de mission auprès du Guichet d'assistance aux projets numériques (GAPN), ENS Paris, EUR Translitteræ 